\title{
MARKOV CELL STRUCTURES FOR EXPANDING MAPS IN DIMENSION TWO
}

\author{
BY
}

F. T. FARRELL AND L. E. JONES ${ }^{1}$

\begin{abstract}
Let $f: M^{2} \rightarrow M^{2}$ be an expanding self-immersion of a closed 2-manifold, then for some positive integer $n, f^{n}$ leaves invariant a cell structure on $M^{2}$. A similar result is true when $M$ is a branched 2-manifold.
\end{abstract}

0. Introduction. Let $K$ be a topological space equipped with a continuous self-map $f$. It is known in many interesting cases that $K$ can be partitioned into cells; i.e., given a cell complex structure. For example, this is possible when $K$ is a smooth manifold. It is rarer for $K$ to support a cell structure with respect to which $f$ is a cellular map (i.e., leaves each skeleton invariant); for instance, this is impossible when $K$ is the circle and $f$ is rotation through an irrational angle.

Consider the situation when $K$ is a closed smooth 2-manifold and $f$ is an expanding immersion of $K$; i.e., $|d f(X)|>|X|$ for all nonzero vectors $X$ tangent to $K$ and some Riemann metric on $K$. In this case, we show (Theorem 2.1 ) there is a positive integer $n$ such that $f^{n}$ (the composite of $f$ with itself $n$-times) is cellular relative to some cell structure on $K$. (We do not know whether $n$ can always be 1 . See [3], [7] and [10] for other recent interesting constructions of invariant sets.)

We prove a similar (slightly weaker) result (Theorem 3.1) for expanding immersions of compact branched 2-manifolds satisfying Axioms 1, 2 and $3^{+}$ of Williams. (See [14] for the basic definitions.) These objects arise in Williams' study [14] of expanding attractors. We hope Theorem 3.1 will be useful in helping to understand 2-dimensional expanding attractors which are apparently more complicated (cf. [5]) than the 1-dimensional case where Williams has a very good theory [13].

Theorems 2.1 and 3.1 are clearly extensions (in a very special setting) of the theory of Markov partitions [1], [12], [2] and [9]. This paper is also an introduction to the results announced in [4].

1. A cellular embedding result. In this section, we formulate and prove a crucial result Lemma 1.1, a strong form of the cellular approximation

Received by the editors April 19, 1978 and, in revised form, October 17, 1978.

AMS (MOS) subject classifications (1970). Primary 58F15.

Key words and phrases. Expanding immersion, branched manifold, cell structure.

${ }^{1}$ Both authors were partially supported by grants from the National Science Foundation. 
theorem for dimension 2. It is used in $\$ 2$ to construct Markov cell structures for expanding maps on 2-manifolds.

Let $\boldsymbol{M}^{2}$ denote a 2-dimensional Riemannian manifold. A special cell structure $C$ in $M^{2}$ is a filtration by closed subsets,

$$
\varnothing=C^{-1} \subset C^{0} \subset C^{1} \subset C^{2}=|C| \subset M^{2},
$$

such that $C^{i}-C^{i-1}(i=0,1,2)$ has finitely many connected components, called the $i$-cells of $C$ with the following properties.

(i) The closure of each $i$-cell $\sigma$ is homeomorphic to $\mathbf{D}^{j}=\{x$ $\left.\in \mathbf{R}^{i}|| x \mid<1\right\}$ via a homeomorphism mapping $\sigma$ onto $\{x \in$ $\left.\mathbf{R}^{i}|| x \mid<1\right\}$. (The closure of a cell is called a closed cell.)

(ii) The intersection of two closed cells is either empty or homeomorphic to $\mathbf{D}^{i}$ (for some $i$ ).

(iii) Each vertex (0-cell) is contained in at least 2 and no more than 3 edges (closed 1-cells).

(iv) Edges are smooth curves.

If $C$ satisfies only properties (i), (ii) and (iii) of (1.1), it is a topological special cell structure.

For a closed subset $A$ of $M^{2}$, we define two types of thickenings $T(A, \varepsilon)$ and $\mathcal{T}(A, C)$ where $\varepsilon>0$ is a real number and $C$ is a special cell structure with $A \subset|C|$;

$$
\begin{aligned}
T(A, \varepsilon) & =\left\{x \in M^{2} \mid d(x, A) \leqslant \varepsilon\right\}, \\
\mathcal{T}(A, C) & =\cup\{\sigma \mid \sigma \text { a closed cell in } C, \sigma \cap A \neq \varnothing\}
\end{aligned}
$$

where $d$ denotes the metric on $M^{2}$.

Choose base points $\left\{P_{\sigma}\right\}$ (where $P_{\sigma} \in \sigma$ ) for the 2-cells $\{\sigma\}$ of $C$ and let $d_{0}>0$ be the smaller of

$$
d\left(\left|C^{1}\right|,\left\{P_{\sigma}\right\}\right) \text { and } \inf \{d(\sigma, \tau) \mid \sigma, \tau \text { closed cells of } C, \sigma \cap \tau=\varnothing\} .
$$

Associate sets $\left\{D_{\sigma}\right\}$, called auxiliary discs, to $\{\sigma\}$ satisfying

(i) $D_{\sigma}$ is homeomorphic to $\mathbf{D}^{2}$,

(ii) $\sigma \subset$ interior $D_{\sigma}$ and

(iii) $D_{\sigma} \subset T\left(\sigma, 10^{-1} d_{0}\right)$.

Assume $M^{2}$ is compact and let $d_{1}>0$ be a number such that, for each $x \in M^{2}$, the exponential map is a diffeomorphism from the disc of radius $d_{1}$ centered at the origin of $T_{x} M^{2}$ (the tangent space to $M^{2}$ at $x$ ) to $T\left(x, d_{1}\right)$. As is customary, let mesh $C$ be the maximum distance between points belonging to a common closed cell in $C$; recall a map $f:|C| \rightarrow|K|$ (between cell structures) is cellular if $f\left(C^{i}\right) \subset K^{i}$ (for each $i$ ). 
LEMMA 1.1. Let $C$ be a special cell structure with $|C|=M^{2}$, mesh $C<$ $(10)^{-1} d_{1}$ and equipped with auxiliary discs $\left\{D_{0}\right\}$; then there exists a number $\varepsilon>0$ such that, given any other special cell structure $K$ with $|K|=M^{2}$ and mesh $K<\varepsilon$, we can construct a cellular homeomorphism $g:|C| \rightarrow|K|$ with $g(\sigma) \subset D_{\sigma}$ for each closed cell $\sigma$ in $C$ and so that $g(\omega)$ contains a vertex of $K$ for each open 1-cell $\omega$ in $C$.

The proof of this result occupies most of $\S 1$. Pick a number $d_{2}>0$ such that $T\left(\sigma, 2 d_{2}\right) \subset D_{\sigma}$ for each closed cell $\sigma$ in $C$ and satisfying the following extra constraint. For each edge $\omega$ in $C$ and vertex $v$ contained in $\omega, \omega$ and the boundary of $T(v, r)$ intersect transversally in a single point provided $0<r \leqslant$ $2 d_{2}$. Consequently, we can smoothly parameterize each edge $\omega$ as a function $\omega:[0,3] \rightarrow M^{2}$ with the following properties

(i) $d \omega(t) / d t \neq 0$ for $t \in[0,3]$,

(ii) $\omega([0,1]) \subset T\left(\omega(0), 3 d_{2} / 2\right)$,

(iii) $\omega([2,3]) \subset T\left(\omega(3), 3 d_{2} / 2\right)$ and

(iv) $\omega((1,2)) \subset M^{2}-\cup\left\{T\left(v, 3 d_{2} / 2\right) \mid v\right.$ a vertex in $\left.C\right\}$.

(Fix such a choice of parameterizations for the remainder of $\$ 1$.)

The construction of $g$ uses the following elementary fact. (Its verification is left as an exercise.)

LEMMA 1.2. If $A$ is a closed connected subset of $M^{2}$ and $K$ is a special cell structure with $|K|=M^{2}$, then $\mathcal{T}(A, K)$ is connected; in fact, any two vertices $v_{0}$ and $v_{1}$ in $\mathcal{T}(A, K)$ can be joined by a simple polygonal arc in $\mathcal{T}(A, K)$.

(A polygonal arc is a concatenation of edges in a complex.)

The $\varepsilon$ posited in Lemma 1.1 is any number smaller than $d_{2} / 3$ satisfying

(i) $T(\omega[0,1], \varepsilon) \subset T\left(\omega(0), 2 d_{2}\right)$,

(ii) $T(\omega[2,3], \varepsilon) \subset T\left(\omega(3), 2 d_{2}\right)$,

(iii) $T(\omega[1,2], \varepsilon) \subset M^{2}-\cup\left\{T\left(v, d_{2}\right) \mid v\right.$ a vertex in $\left.C\right\}$,

(iv) $T\left(\omega_{1}, \varepsilon\right) \cap T\left(\omega_{2}, \varepsilon\right) \subset T\left(v, d_{2}\right)$ if $\omega_{1} \cap \omega_{2}=v$,

where $\omega, \omega_{1}, \omega_{2}$ are (parameterized) edges and $v$ is a vertex of $C$.

First construct $g \mid C^{1}$; for each edge $\omega$, we must determine $g(\omega)$. As an approximation to $g(\omega)$, we construct simple polygonal arcs $\omega^{\prime}:[0,3] \rightarrow$ $\mathcal{T}(\omega, K)$ with the following properties

(i) $\omega^{\prime}[0,1] \subset T\left(\omega(0), 2 d_{2}\right)$,

(ii) $\omega^{\prime}[2,3] \subset T\left(\omega(3), 2 d_{2}\right)$,

(iii) $\omega^{\prime}[1,2] \subset M^{2}-\cup\left\{T\left(v, d_{2}\right) \mid v\right.$ a vertex of $\left.C\right\}$,

(iv) $\omega^{\prime}[0,3] \subset T\left(\omega, 2 d_{2}\right)$ and

(v) if $\omega_{1}(0)=\omega_{2}(0)\left(\omega_{1}(0)=\omega_{2}(3)\right)$, then $\omega_{1}^{\prime}(0)=\omega_{2}^{\prime}(0)\left(\omega_{1}^{\prime}(0)\right.$

$\left.=\omega_{2}^{\prime}(3)\right)$,

where $\omega_{1}, \omega_{2}$ are edges in $C$. 
To construct $\omega^{\prime}$, pick 4 vertices $v_{i}(i=0,1,2,3)$ from $K$ with $v_{i} \in$ $\mathcal{T}(\omega(i), K)$; if $\alpha$ is a second edge in $C$ with $\alpha(0)=\omega(0)$, make the same choice of $v_{0}$ in constructing $\alpha^{\prime}$. (If $\alpha(0)=\omega(3)$, then $v_{0}$ for $\alpha^{\prime}$ should be the $v_{3}$ chosen for $\left.\omega^{\prime}\right)$. Now, using Lemma 1.2 connect successive vertices $v_{i}, v_{i+1}$ by simple polygonal arcs $\gamma_{i}$ in $\mathcal{T}(\omega[i, i+1], K)$; the concatenation of these form a polygonal arc in $\mathcal{T}(\omega[0,3], K)$ connecting $v_{0}$ to $v_{3}$. The result may not be a simple arc; but, it is easy to find subarcs $\gamma_{i}^{\prime}$ of $\gamma_{i}$ which concatenate to form a simple arc $\omega^{\prime}$ connecting $v_{0}$ to $v_{3}$. (Note $v_{1}$ and $v_{2}$ need not be points on $\omega^{\prime}$.) The collection $\left\{\omega^{\prime}\right\}$ thus constructed can be parameterized to satisfy (1.7).

Note, if $\omega_{1}$ and $\omega_{2}$ are nonintersecting edges of $C$, then $\omega_{1}^{\prime}[0,3]$ does not intersect $\omega_{2}^{\prime}[0,3]$. Unfortunately, when $\omega_{1}$ and $\omega_{2}$ are distinct but share a common vertex, possibly $\omega_{1}^{\prime}$ and $\omega_{2}^{\prime}$ meet in more than a common endpoint. However, by an elementary combinatorial argument, this particular collection of $\left\{\omega^{\prime}\right\}$ (constructed above) can be modified to form a new collection $\left\{\omega^{\prime \prime}\right\}$ of simple polygonal arcs having the following properties

(i) $\left\{\omega^{\prime \prime}\right\}$ satisfies (1.7),

(ii) $\omega^{\prime \prime}(t)=\omega^{\prime}(t)$ for $t \in[1,2]$ and

(iii) $\omega^{\prime \prime}[0,3] \cap \alpha^{\prime \prime}[0,3]$ contains at most one point, if $\omega$ and $\alpha$ are distinct edges in $C$.

We leave this argument as an exercise. Hint. The $\left\{\omega^{\prime}\right\}$ can be chosen so that $\cup\left\{\omega^{\prime \prime}[0,3]\right\} \subset \cup\left\{\omega^{\prime}[0,3]\right\}$ but neither of the following pair of equations need be valid

$$
\omega^{\prime \prime}(0)=\omega^{\prime}(0), \quad \omega^{\prime \prime}(3)=\omega^{\prime}(3) .
$$

Figure 1 shows the hinted modification. In it, piecewise smooth curves are used instead of polygonal arcs for purposes of illustration. The dashed lines in the second picture indicate parts of $\cup\left\{\omega_{i}^{\prime}\right\}$ deleted in forming $\left\{\omega_{i}^{\prime \prime}\right\}$; the large circle in both pictures is the boundary of $T\left(x, d_{2}\right)$ where $x=\omega_{1}(0)$ or $\omega_{1}(3)$ as the case requires.

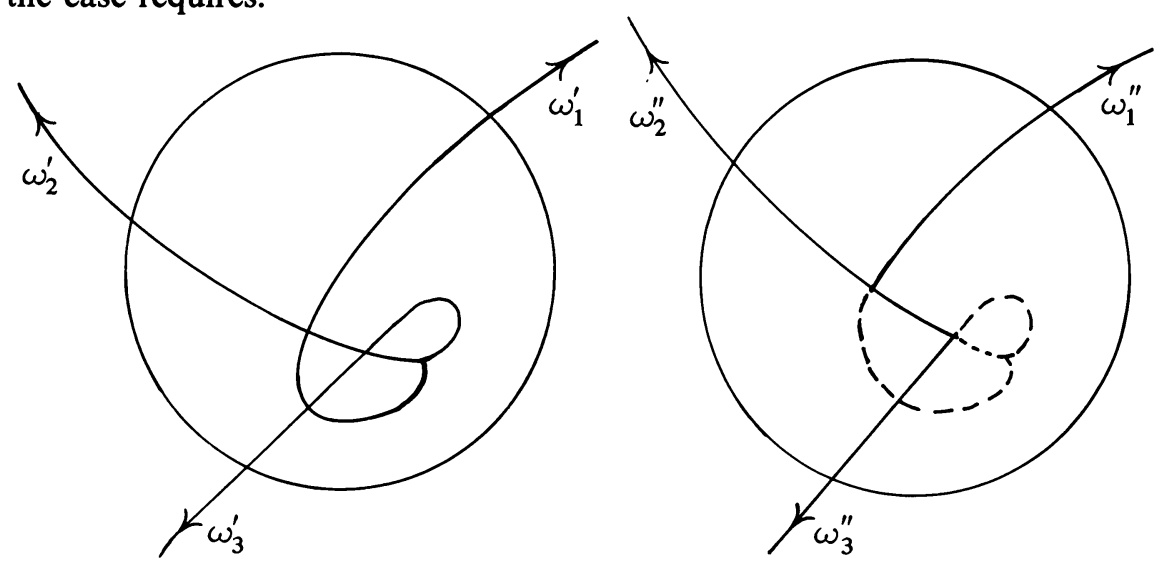

FIGURE 1 
Now define $g \mid C^{1}$ by the formula $g(\omega(t))=\omega^{\prime \prime}(t)$ for $t \in[0,3]$ where $\omega$ is a (parameterized) edge; because of (1.8), this map is an embedding and $g(\omega) \subset D_{\omega}$. (Since mesh $K<d_{2} / 3, g(\omega)$ contains a vertex of $K$ for each open 1-cell $\omega$ in $C$.) In fact, if $\sigma$ is a closed 2-cell and $\partial \sigma$ denotes its boundary, then $g(\partial \sigma) \subset D_{\sigma}$; hence, by Schoenflies' Theorem (cf. [8, p. 175]), $g \mid \partial \sigma$ can be extended to a homeomorphism of $\sigma$ onto the closure of the interior component of $D_{\sigma}-g(\partial \sigma)$. In this way, extend $g \mid C^{1}$ to a cellular map $g$ from $C$ to $K$ with $g(\sigma) \subset D_{\sigma}$ for each closed cell $\sigma$ in $C$. This last fact (cf. (1.4)) implies

$$
g \mid C^{1}: C^{1} \rightarrow M^{2}-\left\{P_{\sigma}\right\}
$$

is homotopic to the inclusion map; hence, by an elementary winding number argument, $g: M^{2} \rightarrow M^{2}$ is a homeomorphism. This completes the proof of Lemma 1.1.

In $\S 3$ a relative version of this result is used. We now formulate it leaving its proof as an exercise.

Drop the compactness constraint on $M^{2}$ and consider the following condition for special cell structures $C$ in $M^{2}$.

For each $x \in|C|$, the exponential map is a diffeomorphism from the disc of radius 10 mesh $C$ centered at the origin of $T_{x} M^{2}$ to $T(x, 10$ mesh $C)$.

LEMMA 1.3. Let $C$ be a special cell structure in $M^{2}$ satisfying (1.10) and equipped with auxiliary discs $\left\{D_{\sigma}\right\}$ (cf. (1.4)); let $U \subset V \subset C$ be subcomplexes such that any closed cell of $C$ which meets $|U|$ is contained in $|V|$. Then there exists a second collection of auxiliary discs $\left\{D_{\sigma}^{\prime}\right\}$ (cf. (1.4)) and a real number $\varepsilon>0$ such that, for any other special cell structure $K$ in $M^{2}$ with mesh $K<\varepsilon$ and $T(|C|$, mesh $C) \subset|K|$ and any cellular embedding $h:|V| \rightarrow|K|$ satisfying $h(\sigma) \subset D_{\sigma}^{\prime}$ for all cells $\sigma$ in $V$, we can construct a cellular embedding $g$ : $|C| \rightarrow|K|$ such that

(i) $g(x)=h(x)$ for $x \in|U|$,

(ii) $g(\sigma) \subset D_{\sigma}$ for all cells $\sigma$ in $C$ and

(iii) $g(\omega)$ contains a vertex of $K$ for each open 1-cell $\omega$ in $C$.

2. Markov cell structures (2-manifold case). Let $M^{2}$ be a closed 2-manifold equipped with a map $f: M^{2} \rightarrow M^{2}$; a Markov cell structure for $\left(M^{2}, f\right)$ is a topological special cell structure $e$ in $M$ (cf. (1.1)) with $|\mathcal{C}|=M$ and such that $f^{n}:|\mathcal{C}| \rightarrow|\mathcal{C}|$ is cellular for some positive integer $n$. This section is devoted to proving the following result.

THEOREM 2.1. If $f: M^{2} \rightarrow M^{2}$ is an expanding endomorphism on a closed 2-manifold, then $\left(M^{2}, f\right)$ has a Markov cell structure.

We note Shub [11] has shown that, under the hypotheses of Theorem 2.1, 
$M^{2}$ is either the torus on the Klein bottle and $f$ is topologically conjugate to a linear expanding map; but we will not use these facts in proving Theorem 2.1.

To prove this result, start by choosing a special cell structure $C$ on $M^{2}$ with $|C|=M^{2}$ and mesh $C<d_{1} / 5$. (See the paragraph preceding the statement of Lemma 1.1 for the definition of $d_{1}$.) Such a complex $C$ can be constructed (for example) by using a dual cell structure to a smooth triangulation of $M^{2}$ having sufficiently small mesh.

Choose a collection of base points $\left\{P_{\sigma}\right\}$ and auxiliary discs $\left\{D_{\sigma}\right\}$ for $C$ (cf. (1.4)) and let $\varepsilon>0$ be the number posited in Lemma 1.1. Let $n$ be a positive integer sufficiently large that

$$
\left|d f^{n}(X)\right|>\varepsilon^{-1}(\text { mesh } C)|X| \text { and } 2|X|
$$

for each nonzero vector $X$ tangent to $M^{2}$ and let $F$ denote $f^{n}$. Since $F$ : $M^{2} \rightarrow M^{2}$ is a covering space, we can form a new special cell structure $K$ with $|K|=M^{2}$ by setting $K^{i}=F^{-1}\left(C^{i}\right)$; note mesh $K<\varepsilon$ because of (2.1). Now, applying Lemma 1.1 to this set up, we obtain a cellular homeomorphism $g:|C| \rightarrow|K|$ with $g(\sigma) \subset D_{\sigma}$ for each closed cell $\sigma$ of $C$. Since $d(x, g(x))<d_{1}$ for all $x \in M^{2}$ and $F$ is expanding, we can (by lifting $g$ via $F^{s}$ ) form a sequence of homeomorphisms $g_{s}: M^{2} \rightarrow M^{2}$ (indexed by the nonnegative integers) having the following properties

$$
\begin{aligned}
& \text { (i) } F g_{s}=g_{s-1} F \text { for } s>0 \text {, } \\
& \text { (ii) } g_{0}=g \text { and } \\
& \text { (iii) } d\left(x, g_{s}(x)\right) \leqslant 2^{-s} d_{1} \text { for all } x \in M^{2} \text {; }
\end{aligned}
$$

in fact, $x$ and $g_{s}(x)$ are in the same component of $F^{-s} T\left(F^{s}(x), d_{1}\right)$. Form the composites $G_{s}=g_{s} g_{s-1} \cdots g_{0}$; because of (2.2), the sequence $G_{s}$ converges uniformly to a continuous function $G: M^{2} \rightarrow M^{2}$ satisfying the equation

$$
F G=G F g \text {. }
$$

Define $\mathcal{C}^{i}$, closed subsets of $M^{2}$, by $\mathcal{C}^{i}=G\left(C^{i}\right)$; because of $(2.3)$ and the fact $F g:|C| \rightarrow|C|$ is cellular, we have

$$
F\left(\bigodot^{i}\right) \subset \bigodot^{i} \text { for all } i \text {. }
$$

To complete the demonstration of Theorem 2.1, it remains to show that the filtration $\mathcal{C}$ (defined above) is a topological special cell structure. (Since $G$ is homotopic to the identity map, we note $|\mathcal{C}|=G\left(M^{2}\right)=M^{2}$.) We will accomplish this by constructing a homeomorphism $G^{\prime}: M^{2} \rightarrow M^{2}$ with $G^{\prime}\left(C^{\prime}\right)=G\left(C^{\imath}\right)$ for all $i$; the construction of $G^{\prime}$ occupies the remainder of this section.

Define a sequence of special cell structures $C(s)$ by the equations $C(s)^{i}=$ $F^{-s}\left(C^{i}\right)$; note $C(0)=C$ and $C(1)=K$. Next, construct a sequence of cellular homeomorphisms $h_{s}: C(s)^{1} \rightarrow C(s)^{1}$ with $h_{s}(\sigma)=\sigma$ for each vertex or edge $\sigma$ of $C(s)$ and having the following property. 
If $\omega$ is an edge of $C(s)$ parameterized by arc length $t$, then $g_{s} h_{s} \omega(\Omega / 2)$ is a vertex of $C(s+1)(\Omega$ denotes the total arc length of $\omega)$ and the derivative of the arc length of $g_{s} h_{s} \omega(t)$ with respect to $t$ exists except when $t=\Omega / 2$ and is constant on $(0, \Omega / 2)$ and $(\Omega / 2, \Omega)$.

It is easy to construct such homeomorphisms using the fact that $g_{s}$ : $|C(s)| \rightarrow|C(s+1)|$ is cellular and $g_{s}(\sigma)$ contains a vertex of $C(s+1)$ for each open 1-cell $\sigma$ of $C(s)$.

Define a sequence of embeddings $H_{i}$ by the equations

$$
H_{i}=g_{i} h_{i} g_{i-1} h_{i-1} \cdots g_{0} h_{0} ;
$$

it follows in a straightforward fashion that this sequence converges uniformly to a continuous function $H: C^{1} \rightarrow M^{2}$ such that $H\left(C^{i}\right)=G\left(C^{i}\right)$ for $i=0$ and 1 .

LEMMA 2.2. The function $H: C^{1} \rightarrow \mathrm{C}^{1}$ is a homeomorphism.

Proof. It remains only to show that $H$ is one-to-one. Let $x$ and $y$ be distinct points in $C^{1}$ and choose a simple piecewise smooth arc $\alpha:[0,1] \rightarrow C^{1}$ with $x=\alpha(a)$ and $y=\alpha(b)$ where $0<a<b<1$ (cf. Lemma 1.2). Because of (2.5) there exists an integer $s>0$ such that the simple arc $H_{s} \alpha$ contains at least four vertices $v_{i}(i=1,2,3,4)$ of $C(s+1)$ with $v_{i}=H_{s} \alpha\left(t_{i}\right)$ where $t_{1}<a<t_{2}<t_{3}<b<t_{4}$; in particular, $H_{s}(x)$ and $H_{s}(y)$ belong to nonintersecting edges $\omega_{x}$ and $\omega_{y}$ of $C(s+1)$. From this remark, it is easily seen (use the defining properties of $\left.h_{i}\right)$ that $H(x) \in \hat{G}_{s+1}\left(\omega_{x}\right)$ and $H(y) \in$ $\hat{G}_{s+1}\left(\omega_{y}\right)$ where $\hat{G}_{s+1}$ is the composite $G G_{s}^{-1}$; hence, Lemma 2.2 is a consequence of the following result.

LEMMA 2.3. If $\sigma_{1}$ and $\sigma_{2}$ are nonintersecting closed cells in $C(s)$, then $\hat{G}_{s}\left(\sigma_{1}\right) \cap \hat{G}_{s}\left(\sigma_{2}\right)=\varnothing$.

Proof. First observe, using Lemma 1.1, 1.4, 2.1, that $G(\sigma) \subset T\left(\sigma, 5^{-1} d_{0}\right)$ for each closed cell $\sigma$ of $C$; also using (2.2), that $F^{s} \hat{G}_{s}=G F^{s}$; hence if $F^{s} \sigma_{1} \cap F^{s} \sigma_{2}=\varnothing$, then $\hat{G}_{s}\left(\sigma_{1}\right) \cap \hat{G}_{s}\left(\sigma_{2}\right)=\varnothing$. On the other hand, if $F^{s} \sigma_{1}$ and $F^{s} \sigma_{2}$ intersect, then $T\left(F^{s} \sigma_{1} \cup F^{s} \sigma_{2}, 5^{-1} d_{0}\right) \subset T\left(x, d_{1}\right)$ for some point $x \in M^{2}$. Since $T\left(x, d_{1}\right)$ is homeomorphic to a closed 2-disc, it is evenly covered by $F^{s}$; in particular, $\sigma_{i} \subset S_{i}(i=1,2)$ where $S_{1}$ and $S_{2}$ are distinct components of $F^{-s} T\left(x, d_{1}\right)$. Finally observe $\sigma_{i} \cup \hat{G}_{s} \sigma_{i} \subset S_{i}(i=1,2)$; hence $\hat{G}_{s} \sigma_{1}$ and $\hat{G}_{s} \sigma_{2}$ cannot intersect.

Because of Lemma 2.2 (and the paragraph preceding it) we define $G^{\prime} \mid C^{1}$ to be $H$. Let $\sigma$ be a closed 2-cell of $C$ with boundary denoted by $\partial \sigma$; then $H(\partial \sigma)$ is contained in the interior of $T\left(P_{\sigma}, d_{1}\right)$ since

$$
H(\partial \sigma)=G(\partial \sigma) \subset G(\sigma) \subset T\left(\sigma, 5^{-1} d_{0}\right)
$$


Since $\partial \sigma$ is homeomorphic to a circle, $T\left(P_{\sigma}, d_{1}\right)$ to a closed 2-disc and $H$ is an embedding, Schoenflies' Theorem allows us to extend $G^{\prime} \mid \partial \sigma$ to a homeomorphism of $\sigma$ to the closure of the interior component of $T\left(P_{\sigma}, d_{1}\right)$ $G^{\prime}(\partial \sigma)$; thus, we extend $G^{\prime} \mid C^{1}$ to all of $M^{2}$.

To complete the demonstration of Theorem 2.1 , it remains to verify that $G^{\prime}$ is a one-to-one function. (Note any one-to-one continuous self-map of a closed connected manifold must be onto.) To do this, first observe that $G^{\prime}(\sigma) \subset G(\sigma)$ for each closed 2-cell $\sigma$ of $C$; this is a consequence of the fact that $G^{\prime} \mid \partial \sigma$ and $G \mid \partial \sigma: \partial \sigma \rightarrow G^{\prime}(\partial \sigma)$ are homotopic (hence $G \mid \partial \sigma$ is essential). Therefore, it suffices to show for all pairs of distinct 2-cells $\sigma_{1}$ and $\sigma_{2}$ in $C$ that the following containment is true

$$
G\left(\sigma_{1}\right) \cap G\left(\sigma_{2}\right) \subset G^{\prime}\left(\sigma_{1} \cap \sigma_{2}\right) .
$$

Let $z \in G\left(\sigma_{1}\right) \cap G\left(\sigma_{2}\right)$; hence, there exist points $x \in \sigma_{1}$ and $y \in \sigma_{2}$ with $G(x)=z=G(y)$; consequently, $d\left(G_{i}(x), G_{i}(y)\right) \rightarrow 0$ as $i \rightarrow \infty$. Since $G_{i}$ is a homeomorphism, there must exist points $x_{i} \in \partial \sigma_{1}$ and $y_{i} \in \partial \sigma_{2}$ such that $G_{i}\left(x_{i}\right) \rightarrow z$ and $G_{i}\left(y_{i}\right) \rightarrow z$ as $i \rightarrow \infty$ (cf. Figure 2); but $\partial \sigma_{i}(i=1,2)$ is compact; hence $z \in G\left(\partial \sigma_{1}\right) \cap G\left(\partial \sigma_{2}\right)$.

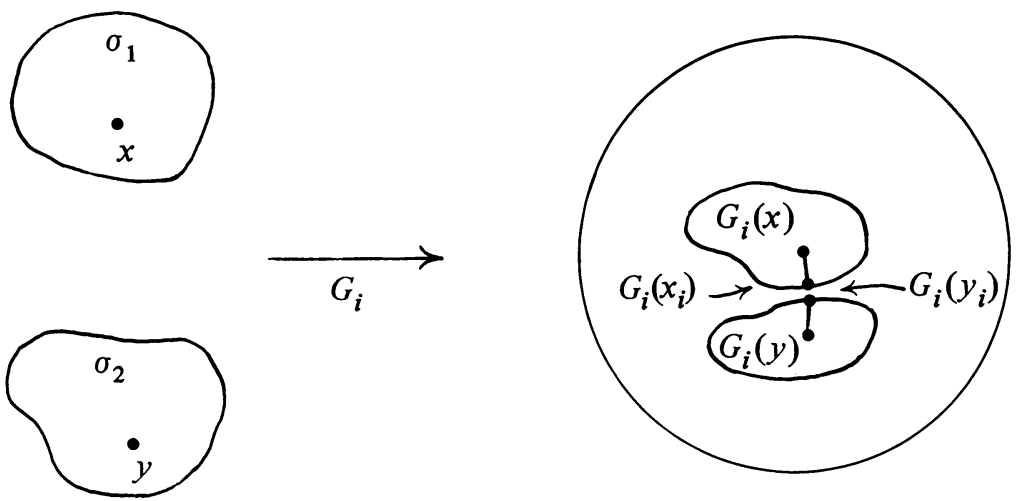

FIGURE 2

Since $G\left(\partial \sigma_{i}\right)=H\left(\partial \sigma_{i}\right)(i=1,2)$ and $H$ is a one-to-one function (Lemma 2.2), we have

$$
z \in H\left(\partial \sigma_{1} \cap \partial \sigma_{2}\right)=G^{\prime}\left(\sigma_{1} \cap \sigma_{2}\right)
$$

which verifies (2.8) and completes the proof of Theorem 2.1.

REMARK 2.4. Let $f: T^{2} \rightarrow T^{2}$, where $T^{2}$ is the torus, be an expanding endomorphism induced by a $2 \times 2$ matrix $A$ with integral entries and whose eigenvalues are real but irrational numbers having distinct absolute values. It is implicit in Franks' paper [6] that a proper closed $f$-invariant subset of $T^{\mathbf{2}}$ cannot contain a $C^{1}$-arc; i.e., a continuously differentiable arc. In particular, 
if

$$
A=\left(\begin{array}{ll}
0 & 11 \\
-1 & 7
\end{array}\right)
$$

then the 1-skeleton of the Markov cell structure constructed in Theorem 2.1 for $\left(T^{2}, f\right)$ cannot contain a $C^{1}$-arc. (See also Bowen's paper [3].)

3. Markov cell structures (branched 2-manifold case). This section is devoted to proving an analogue of Theorem 2.1 in the branched 2-manifold case. (See Williams' paper [14] for the basic definitions and facts concerning branched manifolds.) A cell structure $C$ for a compact branched 2-manifold $M^{2}$ is a filtration of $M^{2}$ by closed subsets

$$
\varnothing=C^{-1} \subset C^{0} \subset C^{1} \subset C^{2}=|C|=M^{2}
$$

such that $C^{i}-C^{i-1}(i=0,1,2)$ has a finite number of components, called $i$-cells, satisfying property (i) in (1.1). If $M^{2}$ is equipped with a map $f$, a Markov cell structure for $\left(M^{2}, f\right)$ is a cell structure $C$ for $M^{2}$ such that $f^{n}$ : $|C| \rightarrow|C|$ is cellular for some positive integer $n$.

THEOREM 3.1. Let $f: M^{2} \rightarrow M^{2}$ be an immersion satisfying Axioms 1, 2 and $3^{+}$of [14] where $M^{2}$ is a compact branched 2-manifold, then $\left(M^{2}, f\right)$ is shift equivalent to a pair $\left(N^{2}, g\right)$ having a Markov cell structure.

The proof of Theorem 3.1 is similar to, but technically more complicated than, that of Theorem 2.1; hence, we sketch it, going into detail only where it differs from the previous argument.

By [14, Lemma 5.6], $\left(M^{2}, f\right)$ is shift equivalent to a pair $\left(N^{2}, g\right)$ where $N^{2}$ is normally branched; hence, we will assume that $M^{2}$ is normally branched. Let $\beta M$ denote the branch set of $M^{2}$; as a consequence of $[14, \S 8], M^{2}$ contains a 2-dimensional compact submanifold (with boundary) $A$ and a 2-dimensional compact branched submanifold (with boundary) $B^{\prime}$ satisfying

$$
\begin{aligned}
& \text { (i) interior } A \cup \text { interior } B^{\prime}=M^{2} \text { and } \\
& \text { (ii) } \beta M \subset \text { interior } B^{\prime} \cap\left(M^{2}-A\right) \text {; }
\end{aligned}
$$

furthermore, there is a compact 2-manifold $B$ (with boundary), a surjective immersion $p: B^{\prime} \rightarrow B$ which maps $\beta M$ homeomorphically onto $p(\beta M)$, and an immersion $\varphi: B \rightarrow M^{2}$ with $f(x)=\varphi p(x)$ for all $x \in B^{\prime}$. By deleting short collar neighborhoods from $A, B$ and $B^{\prime}$, we obtain compact submanifolds $A_{i}$, $B_{i}$ and $B_{i}^{\prime}(i=-1,0,1)$ with

$$
\begin{aligned}
& A_{-1} \subset \text { interior } A_{0} \subset \text { interior } A_{1} \subset \text { interior } A \text { and } \\
& B_{-1} \subset \text { interior } B_{0} \subset \text { interior } B_{1} \subset \text { interior } B
\end{aligned}
$$

such that $B_{i}^{\prime}=p^{-1} B_{i}$ and (3.2) is satisfied when $A$ and $B^{\prime}$ are replaced by $A_{-1}$ and $B_{-1}^{\prime}$. We also assume that the Riemannian metrics on $M^{2}, A, B^{\prime}$ and $B$ fit together consistently; i.e., $A$ and $B^{\prime}$ have the metrics induced from $M^{2}$ 
and $d p: T B^{\prime} \rightarrow T B$ preserves the Riemann metric.

A (topological) branched cell structure $\mathscr{B}$ for $M^{2}$ is a filtration by closed subsets

$$
\varnothing=\mathscr{B}^{-1} \subset \mathscr{B}^{0} \subset \mathscr{B}^{1} \subset \mathscr{B}^{2}=|\mathscr{B}|=M^{2}
$$

such that there exist (topological) special cell structures $C$ in the interior of $A$ and $K$ in the interior of $B$ satisfying the following conditions

(i) $\mathscr{B}^{i}=C^{i} \cup p^{-1} K^{i}$,

(ii) $A_{-1} \subset|C|, B_{-1} \subset|K|$ and

(iii) $|C| \cap p^{-1}|K|$ is a subcomplex of $C$; each cell of which

maps homeomorphically via $p$ onto a cell of $K$.

The components of $\mathscr{B}^{i}-\mathscr{B}^{i-1}$ are (in general) not cells when they intersect $\beta M^{2}$. A map $f:|\mathscr{B}| \rightarrow|\mathscr{B}|$ is cellular if $f\left(\mathscr{B}^{i}\right) \subset \mathscr{B}^{i}$ (for all $i$ ). We will derive Theorem 3.1 directly from the following result.

LEMMA 3.2. There is a topological branched cell structure $\mathscr{B}$ for $M^{2}$ of arbitrarily small mesh such that $f^{n}:|\Re| \rightarrow|\Re|$ is a cellular map for some positive integer $n$.

Before proving this lemma, we use it together with the collapsing technique introduced by Williams (cf. [14, Lemmas 2.2 and 5.4]) to complete the demonstration of Theorem 3.1. Let $K$ be the topological special cell structure for $B$ posited in (3.4). Since $\mathscr{B}$ can be constructed with arbitrarily small mesh, we can assume that $K$ has a subcomplex $K_{0}$ such that

$$
p\left(\beta M^{2}\right) \subset \text { interior }\left|K_{0}\right| \subset \text { interior } B_{-1} \text {. }
$$

Form $N^{2}$ by collapsing $p^{-1}\left|K_{0}\right|$ under the immersion $p: B^{\prime} \rightarrow B$ and let $g$ : $N^{2} \rightarrow N^{2}$ be the immersion induced from $f$, then $\left(N^{2}, g\right)$ is shift equivalent to $\left(M^{2}, f\right)$ and $\mathscr{B}$ induces a cell structure on $N^{2}$ (via the canonical quotient map) with respect to which $g^{n}$ is cellular; i.e., $\left(N^{2}, g\right)$ has a Markov cell structure.

REMARK 3.3. The Markov cell structure constructed above may fail to satisfy property (ii) in (1.1); for instance, if $K_{0}$ is not a "full" subcomplex in $K$, this is possible. But, by constructing more carefully, we believe this property can also be satisfied.

It remains to prove Lemma 3.2 ; we start by constructing a branched cell structure $\mathscr{B}(0)$ for $M^{2}$ of arbitrarily small mesh. First, triangulate $M^{2}$ by a smooth triangulation $\mathcal{T}_{1}$ (with arbitrarily small mesh) so that $B^{\prime}$ is a subcomplex with its triangulation induced via $p^{-1}$ from a smooth triangulation $\mathcal{F}_{2}$ of $B$ (cf. [14, Lemma 5.7]). Let $\mathscr{F}_{1}$ and $\mathscr{F}_{2}$ be subcomplexes consisting of all closed simplices from $\mathcal{T}_{1}$ and $\mathcal{T}_{2}$, respectively, which meet $A_{0}$ and $B_{1}$, respectively; if mesh $\mathcal{T}_{1}$ is sufficiently small, then

$$
\left|\mathscr{F}_{1}\right| \subset \text { interior } A_{1} \text { and }\left|\mathscr{F}_{2}\right| \subset \text { interior } B \text {. }
$$


Let $C$ and $K$ be the dual cell complexes to $\mathscr{F}_{1}$ and $\mathscr{F}_{2}$, respectively; then $C, K$ satisfy conditions (ii) and (iii) of (3.4) and hence determine via formula (i) of (3.4) a branched cell structure $\mathscr{B}(0)$ for $M^{2}$ with arbitrarily small mesh. (Note also $B_{1} \subset|K|$ and $|C| \subset A_{1}$.)

We assume that

$$
|d f(X)|>2|X|
$$

for each vector $X$ tangent to $M^{2}$. (To do this, we may have to replace $f$ by a power of itself.) Define a second branched cell structure $\mathscr{B}(1)$ for $M^{2}$ by letting $\mathscr{B}(1)^{i}=f^{-1}\left(\mathscr{B}(0)^{i}\right)$. (For this to be a branched cell structure, mesh $\mathscr{B}(0)$ must be sufficiently small.) There are special cell structures $C(1)$ in the interior of $A$ and $K(1)$ in the interior of $B$ satisfying (3.4) (with $\mathscr{B}, C$ and $K$ replaced by $\mathscr{B}(1), C(1)$ and $K(1)$ ) and having the following properties

(i) $C(1)^{i} \subset \mathscr{B}(1)^{i}$ and $K(1)^{i} \subset \varphi^{-1}\left(\mathscr{B}(0)^{i}\right)$;

(ii) $T(|C|$, mesh $\Re(0)) \subset|C(1)|$ and $T(|K|$, mesh $\mathscr{B}(0)) \subset$ $|K(1)|$.

(In order to satisfy property (ii), mesh $\mathscr{B}(0)$ must again be sufficiently small.)

Let $\left\{P_{\sigma}\right\},\left\{D_{\sigma}\right\}$ be base points and auxiliary discs for $C$; let $V=p^{-1}|K| \cap$ $|C|$ and $U=p^{-1} \mathscr{T}\left(B_{0}, K\right) \cap|C|$. If mesh $\mathscr{B}(0)$ is sufficiently small, then $U \subset V \subset C$ satisfy the first hypothesis of Lemma 1.3 ; let $\left\{D_{\sigma}^{\prime}\right\}$ be the auxiliary discs posited there. Replacing these by smaller discs (if necessary), we may assume there are auxiliary discs $\left\{\hat{D}_{\tau}\right\}$ for $K$ such that $p\left(D_{\sigma}^{\prime}\right)=\hat{D}_{\tau}$ for each closed cell $\sigma$ of $V$ and $\tau$ of $K$ with $p(\sigma)=\tau$. Also let $\left\{\hat{P}_{\tau}\right\}$ be a collection of base points for $K$ such that $p\left(P_{\sigma}\right)=\hat{P}_{\tau}$ for 2-cells $\sigma$ of $V$ and $\tau$ of $K$ with $p(\sigma)=\tau$. Replacing $f$ by a high power of itself (if necessary), we can assume that mesh $\mathscr{B}(1)$ is small enough so that we can apply Lemma 1.3 (twice) to obtain cellular embeddings $\hat{g}:|K| \rightarrow|K(1)|$ and $g:|C| \rightarrow|C(1)|$ with the following properties

(i) $p g(x)=\hat{g} p(x)$ for $x \in|C| \cap p^{-1}|K(0)|$ where $K(0)=$ $\mathcal{T}\left(B_{0}, K\right)$;

(ii) $g(\sigma) \subset D_{\sigma}$ and $\hat{g}(\tau) \subset \hat{D}_{\tau}$ where $\sigma$ and $\tau$ are closed cells in $C$ and $K$, respectively.

Of course, mesh $\mathscr{B}(0)$ must be small enough that Lemma 1.3 applies; in particular, $T(x, 5 c)$ should be homeomorphic to a closed 2-disc for both $x \in A_{0}$ and $x \in B_{0}$ where $c=2$ mesh $\mathscr{B}(0)$; also, assume $T\left(A_{1}, c\right) \subset A$ and $T\left(B_{1}, c\right) \subset B$. Now, define inductively a sequence of embeddings $g_{s}: A_{1} \rightarrow A$ and $\hat{g}_{s}: B_{1} \rightarrow B$ as follows. If $x \in A_{1}$ and $f(x) \in A_{0}$ (respectively, $f(x) \in B_{0}^{\prime}$ ), let $g_{s}(x)$ be the unique point in the same component of $f^{-1} T(f(x), c)$, $\left(f^{-1} p^{-1} T(p f(x), c)\right)$ as $x$ such that 


$$
f g_{s}(x)=g_{s-1} f(x), \quad\left(p f g_{s}(x)=\hat{g}_{s-1} p f(x)\right) ;
$$

if $x \in B_{1}$ and $y=\varphi(x) \in A_{0}$ (respectively, $y \in B_{0}^{\prime}$ ), let $\hat{g}_{s}(x)$ be the unique point in the same component of $\varphi^{-1} T(y, c),\left(\varphi^{-1} p^{-1} T(p(y), c)\right)$ as $x$ such that

$$
\varphi \hat{g}_{s}(x)=g_{s-1}(y), \quad\left(p \varphi \hat{g}_{s}(x)=\hat{g}_{s-1} p(y)\right) .
$$

(When $s=1$ use $g$ and $\hat{g}$ in formulas 3.10.1, 3.10.2 in place of $g_{0}$ and $\hat{g}_{0}$, respectively.) Note the following analogue of 3.9(i) is true

$$
p g_{s}(x)=\hat{g}_{s} p(x) \text { for } x \in A_{1} \cap B_{1}^{\prime} \text {. }
$$

Next, form the composite embeddings $G_{s}:|C(0)| \rightarrow A$ where $C(0)=$ $\mathcal{T}\left(A_{0}, C\right)$ and $\hat{G}_{s}:|K(0)| \rightarrow B$ defined by the equations

$$
\begin{aligned}
& G_{s}(x)=g_{s} g_{s-1} \cdots g_{1} g(x) \text { for } x \in|C(0)| \quad \text { and } \\
& \hat{G}_{s}(y)=\hat{g}_{s} \hat{g}_{s-1} \cdots \hat{g}_{1} \hat{g}(y) \text { for } y \in|K(0)| .
\end{aligned}
$$

These sequences converge uniformly to continuous functions $G:|C(0)| \rightarrow A$ and $\hat{G}:|K(0)| \rightarrow B$ satisfying

$$
p G(x)=\hat{G} p(x) \text { for } x \in|C(0)| \cap p^{-1}|K(0)| \text {. }
$$

Define filtrations $\mathcal{C}$ and $\mathcal{K}$ by the equations

$$
\mathfrak{C}^{i}=G\left(C(0)^{i}\right) \text { and } \mathscr{K}^{i}=\hat{G}\left(K(0)^{i}\right) ;
$$

although $G$ and $\hat{G}$ need not be embeddings, arguing as in $\$ 2$, it can be shown that $\mathcal{C}$ and $\mathscr{K}$ are topological special cell structures in the interiors of $A$ and $B$, respectively. Also, conditions (ii) and (iii) of (3.4) are satisfied when $C$ and $K$ are replaced by $\mathcal{C}$ and $\mathscr{K}$; hence, letting $\mathscr{B}^{i}=\mathcal{C}^{i} \cup p^{-1} \mathcal{K}^{i}$, we obtain a topological branched cell structure $\mathscr{B}$ for $M^{2}$ and, using (3.10.1), (3.10.2), it can be shown that $f:|\mathscr{B}| \rightarrow|\mathscr{B}|$ is cellular. This completes the proof of Lemma 3.2.

\section{REFERENCES}

1. R. L. Adler and B. Weiss, Entropy, a complete metric invariant for automorphisms of the torus, Proc. Nat. Acad. Sci. U.S.A. 57 (1967), 1573-1576.

2. R. Bowen, Markov partitions for Axiom A diffeomorphisms, Amer. J. Math. 92 (1970), 725-747.

3. Markov partitions are not smooth (to appear).

4. F. T. Farrell and L. E. Jones, Markov cell structures, Bull. Amer. Math. Soc. 83 (1977), 739-740.

5. __ New attractors in hyperbolic dynamics, J. Differential Geometry (to appear).

6. J. M. Franks, Invariant sets of hyperbolic toral automorphisms, Amer. J. Math. 99 (1977), 1089-1095.

7. S. G. Hancock, Orbits and paths under hyperbolic toral automorphisms (to appear).

8. J. G. Hocking and G. S. Young, Topology, Addison-Wesley, London, 1961.

9. K. Krzyżewski, On connection between expanding mappings and Markov chains, Bull. Acad. Polon. Sci. Sér. Sci. Math. Astronom. Phys. (4) 19 (1971), 291-293. 
10. F. Przytycki, Construction of invariant sets for Anosov diffeomorphisms and hyperbolic attractors (to appear).

11. M. Shub, Endomorphisms of compact differentiable manifolds, Amer. J. Math. 91 (1969), 175-199.

12. I. G. Sinai, Markov partitions and C-diffeomorphisms, Funkcional Anal. i Priložen. 2 (1) (1968), 64-89.

13. R. F. Williams, Classification of 1-dimensional attractors, Proc. Sympos. Pure Math., vol. 14, Amer. Math. Soc., Providence, R. I., 1970, pp. 341-361.

14. , Expanding attractors, Inst. Hautes Ėtudes Sci. Publ. Math. 43 (1974), 169-203.

Department of Mathematics, Pennsyluania State University, University Park, PennSYLVANIA 16802

Department of Mathematics, SUNY Center at Stony Brook, Stony Brook, New York 11794

Current address (F. T. Farrell): Department of Mathematics, University of Michigan, Ann Arbor, Michigan 48109

Current address (L. E. Jones): Department of Mathematics, Yale University, New Haven, Connecticut 06520 